Supplement of Atmos. Chem. Phys., 18, 16863-16883, 2018

https://doi.org/10.5194/acp-18-16863-2018-supplement

(c) Author(s) 2018. This work is distributed under

the Creative Commons Attribution 4.0 License.

(c) (1)

Supplement of

\title{
Impacts of physical parameterization on prediction of ethane concentrations for oil and gas emissions in WRF-Chem
}

Maryam Abdi-Oskouei et al.

Correspondence to: Maryam Abdi-Oskouei (maryam-abdioskouei@uiowa.edu)

The copyright of individual parts of the supplement might differ from the CC BY 4.0 License. 


\section{S1. Definition of statistical measures}

For quantitative comparison between the simulations we used statistical measures including correlation coefficient (R), root mean square error (RMSE), mean absolute error (MAE), mean bias (MB), and normalized mean bias (NMB). Definitions of these metrics can be found below:

$$
\begin{aligned}
& R=\frac{\overline{\left(C_{o}-\overline{C_{o}}\right)\left(C_{p}-\overline{C_{p}}\right)}}{\sigma_{C p} \sigma_{C o}} \\
& R M S E=\sqrt{\frac{\sum_{i=1}^{n}\left(C_{p_{i}}-C_{o_{i}}\right)^{2}}{n}} \\
& M A E=\frac{1}{n} \sum_{i=1}^{n}\left|C_{p_{i}}-C_{o_{i}}\right| \\
& M B=\frac{1}{n} \sum_{i=1}^{n}\left(C_{p_{i}}-C_{o_{i}}\right) \\
& N M B=\frac{\left(\overline{C_{p}}-\overline{C_{o}}\right)}{\overline{C_{o}}} \times 100 \%
\end{aligned}
$$

Where $\mathrm{C}_{\mathrm{o}}$ is the observation value, $\mathrm{C}_{\mathrm{p}}$ is the model value, $\sigma$ is the standard deviation, $\bar{C}$ is the mean value, and $\mathrm{n}$ is total number of observation points 
Table S1 Conversion table used to map species from NEI-2011 emission inventory to RACM chemical mechanism in and MADE/SORGAM aerosol module

\begin{tabular}{|c|c|c|c|}
\hline $\begin{array}{l}\text { Emission } \\
\text { inventory name }\end{array}$ & $\begin{array}{l}\text { WRF-Chem } \\
\text { name }\end{array}$ & Weight & Species name \\
\hline $\mathrm{CO}$ & e_co & 1.00 & Carbon monoxide \\
\hline NOX & e_no & 1.00 & Nitrogen Oxides (NO or NO2) \\
\hline $\mathrm{SO} 2$ & e_so2 & 1.00 & Sulfur dioxide \\
\hline NH3 & e_nh3 & 1.00 & Ammonia \\
\hline $\mathrm{HC} 01$ & e $\_$ch4 & 1.00 & Methane \\
\hline $\mathrm{HCO} 2$ & e eth & 1.00 & Ethane $\mathrm{kOH}<500 / \mathrm{ppm} / \mathrm{min}$ \\
\hline $\mathrm{HC} 03$ & e_hc3 & 1.00 & $\begin{array}{l}\text { Alkane } 500<\mathrm{kOH}<2500 \\
\text { exclude }(\mathrm{C} 3 \mathrm{H} 8, \mathrm{C} 2 \mathrm{H} 2 \text {,ethanol,acids })\end{array}$ \\
\hline $\mathrm{HC} 04$ & e hc3 & 1.11 & Alkane $2500<\mathrm{kOH}<5000$ exlude(butanes) \\
\hline $\mathrm{HC} 05$ & e_hc5 & 0.97 & Alkane $5000<\mathrm{kOH}<10000$ exlude(pentanes) \\
\hline $\mathrm{HC} 06$ & e hc8 & 1.00 & Alkane $\mathrm{kOH}>10000$ exclude(ethylene glycol) \\
\hline $\mathrm{HC} 07$ & e_ol2 & 1.00 & Ethylene \\
\hline $\mathrm{HC} 08$ & e_olt & 1.00 & Alkene $\mathrm{kOH}<20000 / \mathrm{ppm} / \mathrm{min}$ \\
\hline $\mathrm{HC} 09$ & e oli & 1.00 & $\begin{array}{l}\text { Alkene } \mathrm{kOH}>20000 / \mathrm{ppm} / \mathrm{min} \\
\text { exclude(dienes,styrenes) }\end{array}$ \\
\hline $\mathrm{HC} 10$ & e iso & 1.00 & Isoprene \\
\hline $\mathrm{HC} 12$ & e tol & 1.00 & $\begin{array}{l}\text { Aromatic } \mathrm{kOH}<20000 / \mathrm{ppm} / \mathrm{min} \text { exclude(benzene and } \\
\text { toluene) }\end{array}$ \\
\hline $\mathrm{HC} 13$ & e_xyl & 1.00 & Aromatic $\mathrm{kOH}>20000 / \mathrm{ppm} / \mathrm{min}$ exclude(xylenes) \\
\hline $\mathrm{HC} 14$ & e hcho & 1.00 & Formaldehyde \\
\hline $\mathrm{HC} 15$ & e_ald & 1.00 & Acetaldehyde \\
\hline $\mathrm{HC} 16$ & e_ald & 1.00 & Higher aldehydes \\
\hline $\mathrm{HC} 17$ & e_ald & 1.00 & Benzaldehyde \\
\hline $\mathrm{HC} 18$ & e ket & 0.33 & Acetone \\
\hline $\mathrm{HC} 19$ & e ket & 1.61 & Methylethyl ketone \\
\hline $\mathrm{HC} 20$ & e_ket & 1.61 & PRD2 SAPRAC species (aromatic ketones) \\
\hline $\mathrm{HC} 21$ & e hc3 & 0.40 & Methanol \\
\hline $\mathrm{HC} 22$ & e_ald & 1.00 & Glyoxal \\
\hline $\mathrm{HC} 23$ & e_ald & 1.00 & Methylglyoxal \\
\hline $\mathrm{HC} 24$ & e_ald & 1.00 & Biacetyl \\
\hline $\mathrm{HC} 25$ & e csl & 1.00 & Phenols \\
\hline $\mathrm{HC} 26$ & e_csl & 1.00 & Cresols \\
\hline $\mathrm{HC} 27$ & e ald & 0.50 & Methacrolein \\
\hline $\mathrm{HC} 27$ & e olt & 0.50 & Methacrolein \\
\hline $\mathrm{HC} 28$ & e_ket & 0.50 & Methylvinyl ketone \\
\hline
\end{tabular}




\begin{tabular}{|c|c|c|c|}
\hline $\mathrm{HC} 28$ & e olt & 0.50 & Methylvinyl ketone \\
\hline $\mathrm{HC} 29$ & e ket & 1.00 & IPRD SAPRAC species ( $>\mathrm{C} 4$ unsaturated aldehydes) \\
\hline $\mathrm{HC} 31$ & e_ora2 & 1.00 & Acetic Acid \\
\hline $\mathrm{HC} 32$ & e ora2 & 1.00 & $>\mathrm{C} 2$ Acids (SAPRC PACD species) \\
\hline $\mathrm{HC} 33$ & e_csl & 1.00 & Xylenols (SAPRC-11 species) \\
\hline $\mathrm{HC} 34$ & e csl & 1.00 & Catechols (SAPRC-11 species) \\
\hline $\mathrm{HC} 36$ & e_olt & 1.00 & Propylene \\
\hline $\mathrm{HC} 37$ & e he3 & 0.40 & Acetylene \\
\hline $\mathrm{HC} 38$ & e tol & 0.29 & Benzene \\
\hline HC39 & e hc3 & 1.11 & Butanes \\
\hline $\mathrm{HC} 40$ & e hes & 0.97 & Pentanes \\
\hline $\mathrm{HC} 41$ & e tol & 1.00 & Toluene \\
\hline $\mathrm{HC} 42$ & e_xyl & 1.00 & m-Xylene \\
\hline $\mathrm{HC} 43$ & e $x y l$ & 1.00 & p-Xylene \\
\hline $\mathrm{HC} 44$ & e $x y l$ & 1.00 & o-Xylene \\
\hline $\mathrm{HC} 45$ & e $\mathrm{hc} 3$ & 0.57 & Propane \\
\hline $\mathrm{HC} 46$ & e oli & 1.00 & Dienes \\
\hline $\mathrm{HC} 47$ & e_olt & 1.00 & Styrenes \\
\hline $\mathrm{HC} 47$ & e tol & 1.00 & Styrenes \\
\hline $\mathrm{HC} 48$ & e_hc3 & 1.20 & Ethanol \\
\hline $\mathrm{HC} 49$ & e hc 8 & 1.14 & Ethylene Glycol \\
\hline PM01 & e $\_$pm $25 \mathrm{i}$ & 0.20 & Unspeciated primary PM2.5 - nuclei mode \\
\hline PM01 & e $p m 25 \mathrm{j}$ & 0.80 & Unspeciated primary PM2.5 - accumulation mode \\
\hline $\mathrm{PM} 02$ & e_so4i & 0.20 & Sulfate PM2.5 - nuclei mode \\
\hline $\mathrm{PM} 02$ & e so $4 \mathrm{j}$ & 0.80 & Sulfate PM2.5 - accumulation mode \\
\hline PM03 & e no3i & 0.20 & Nitrate PM2.5 - nuclei mode \\
\hline PM03 & e_no3j & 0.80 & Nitrate PM2.5 - accumulation mode \\
\hline PM04 & e_orgi & 0.20 & Organic Carbon PM2.5 - nuclei mode \\
\hline PM04 & e_orgj & 0.80 & Organic Carbon PM2.5 - accumulation mode \\
\hline PM05 & e_eci & 0.20 & Elemental Carbon PM2.5 - nuclei mode \\
\hline PM05 & e_ecj & 0.80 & Elemental Carbon PM2.5 - accumulation mode \\
\hline PM10-PRI & e pm10 & 1.00 & Unspeciated Primary PM10 \\
\hline
\end{tabular}


Table S2. Summary of model performance in capturing temperature at BAO $10 \mathrm{~m}$ and $300 \mathrm{~m}$ during Aug 1-15, 2014

\begin{tabular}{|c|c|c|c|c|c|c|c|c|c|c|}
\hline \multirow{2}{*}{$\begin{array}{l}T(C)- \\
10 m\end{array}$} & \multirow[b]{2}{*}{ OBS } & \multicolumn{3}{|c|}{ PBL } & \multicolumn{2}{|c|}{ Met IC and BC } & \multicolumn{2}{|c|}{ Initialization } & \multicolumn{2}{|c|}{$\begin{array}{l}\text { Horizontal } \\
\text { resolution }\end{array}$} \\
\hline & & PBL1 & PBL2 & PBL3 & Met5 & Met6 & Init4 & Init5 & Hor5 & $\begin{array}{l}\text { Hor5- } \\
12 \mathrm{~km} \\
\end{array}$ \\
\hline Mean & 21.67 & 22.40 & 20.95 & 21.20 & 24.06 & 23.44 & 21.59 & 24.06 & 24.06 & 24.08 \\
\hline $\mathbf{R}$ & & 0.89 & 0.89 & 0.89 & 0.86 & 0.89 & 0.71 & 0.86 & 0.86 & 0.88 \\
\hline RMSE & & 2.05 & 2.03 & 2.01 & 3.25 & 2.63 & 2.99 & 3.25 & 3.25 & 3.18 \\
\hline MAE & & 1.56 & 1.62 & 1.59 & 2.60 & 2.05 & 2.30 & 2.60 & 2.60 & 2.53 \\
\hline MB & & 0.74 & -0.72 & -0.46 & 2.40 & 1.77 & -0.08 & 2.40 & 2.40 & 2.41 \\
\hline NMB & & $3.4 \%$ & $-3.3 \%$ & $-2.1 \%$ & $11.1 \%$ & $8.2 \%$ & $-0.4 \%$ & $11.1 \%$ & $11.1 \%$ & $11.1 \%$ \\
\hline $\begin{array}{l}\text { T (C) - } \\
\text { 300m }\end{array}$ & OBS & PBL1 & PBL2 & PBL3 & Met5 & Met6 & Init4 & Init5 & Hor5 & $\begin{array}{l}\text { Hor5- } \\
12 \text { km }\end{array}$ \\
\hline Mean & & 21.91 & 20.95 & 21.30 & 23.58 & 22.89 & 20.31 & 23.58 & 23.58 & 23.52 \\
\hline $\mathbf{R}$ & & 0.76 & 0.75 & 0.72 & 0.74 & 0.78 & 0.57 & 0.74 & 0.74 & 0.75 \\
\hline RMSE & & 2.16 & 2.14 & 2.10 & 2.79 & 2.27 & 3.09 & 2.79 & 2.79 & 2.80 \\
\hline MAE & & 1.69 & 1.73 & 1.68 & 2.24 & 1.76 & 2.45 & 2.24 & 2.24 & 2.21 \\
\hline MB & & 0.23 & -0.73 & -0.38 & 1.90 & 1.22 & -1.37 & 1.90 & 1.90 & 1.85 \\
\hline NMB & & $1.1 \%$ & $-3.4 \%$ & $-1.8 \%$ & $8.8 \%$ & $5.6 \%$ & $-6.3 \%$ & $8.8 \%$ & $8.8 \%$ & $8.5 \%$ \\
\hline
\end{tabular}


Table S3. Summary of model performance in capturing relative humidity $(\mathrm{RH})$ at BAO $10 \mathrm{~m}$ and 300m during Aug 1-15, 2014

\begin{tabular}{|c|c|c|c|c|c|c|c|c|c|c|}
\hline \multirow[b]{2}{*}{ RH (\%)-10m } & \multirow[b]{2}{*}{ OBS } & \multicolumn{3}{|c|}{ PBL } & \multicolumn{2}{|c|}{ Met IC and BC } & \multicolumn{2}{|c|}{ Initialization } & \multicolumn{2}{|c|}{$\begin{array}{l}\text { Horizontal } \\
\text { resolution }\end{array}$} \\
\hline & & PBL1 & PBL2 & PBL3 & Met5 & Met6 & Init4 & Init5 & Hor5 & $\begin{array}{l}\text { Hor5- } \\
12 \mathrm{~km}\end{array}$ \\
\hline Mean & 46.47 & 46.85 & 57.59 & 55.78 & 32.65 & 39.87 & 59.36 & 32.65 & 32.65 & 32.89 \\
\hline $\mathbf{R}$ & & 0.78 & 0.69 & 0.73 & 0.63 & 0.64 & 0.53 & 0.63 & 0.63 & 0.71 \\
\hline RMSE & & 10.89 & 16.90 & 15.13 & 19.13 & 14.95 & 22.33 & 19.13 & 19.13 & 18.15 \\
\hline MAE & & 8.45 & 14.38 & 12.86 & 15.01 & 11.31 & 18.10 & 15.01 & 15.01 & 14.43 \\
\hline MB & & 0.38 & 11.12 & 9.31 & -13.81 & -6.60 & 12.90 & -13.51 & -13.51 & -13.58 \\
\hline NMB & & $0.8 \%$ & $23.9 \%$ & $20.0 \%$ & $-29.7 \%$ & $-14.2 \%$ & $27.7 \%$ & $-29.7 \%$ & $-29.7 \%$ & $-29.2 \%$ \\
\hline $\begin{array}{l}\text { RH (\%)- } \\
\text { 300m }\end{array}$ & OBS & PBL1 & PBL2 & PBL3 & Met5 & Met6 & Init4 & Init5 & Hor5 & $\begin{array}{l}\text { Hor5- } \\
12 \mathrm{~km} \\
\end{array}$ \\
\hline Mean & 38.70 & 43.63 & 51.45 & 48.25 & 31.27 & 38.55 & 59.06 & 31.27 & 31.27 & 31.94 \\
\hline $\mathbf{R}$ & & 0.64 & 0.59 & 0.48 & 0.53 & 0.52 & 0.41 & 0.53 & 0.53 & 0.57 \\
\hline RMSE & & 13.06 & 17.92 & 15.25 & 12.66 & 11.14 & 28.39 & 12.66 & 12.66 & 12.11 \\
\hline MAE & & 9.92 & 14.78 & 12.77 & 9.73 & 8.60 & 23.19 & 9.73 & 9.73 & 9.29 \\
\hline MB & & 4.93 & 12.75 & 9.55 & -7.43 & -0.15 & 20.36 & -7.43 & -7.43 & -6.76 \\
\hline NMB & & $12.7 \%$ & $32.9 \%$ & $24.7 \%$ & $-19.2 \%$ & $-0.4 \%$ & $52.6 \%$ & $-19.2 \%$ & $-19.2 \%$ & $-17.5 \%$ \\
\hline
\end{tabular}


Table S4 Summary of model performance in capturing wind speed and direction at BAO 10m during Aug 1-15, 2014

\begin{tabular}{|c|c|c|c|c|c|c|c|c|c|c|c|}
\hline \multirow{2}{*}{\multicolumn{2}{|c|}{ Day - 10 m }} & \multirow{3}{*}{\begin{tabular}{|l|} 
OBS \\
2.46
\end{tabular}} & \multicolumn{3}{|c|}{ PBL } & \multicolumn{2}{|c|}{ Met } & \multicolumn{2}{|c|}{ Init } & \multicolumn{2}{|c|}{ Horizontal Res. } \\
\hline & & & PBL1 & PBL2 & PBL3 & Met5 & Met6 & Init4 & Init5 & Hor5 & $\begin{array}{l}\text { Hor5- } \\
12 \mathrm{~km} \\
\end{array}$ \\
\hline \multirow{2}{*}{ 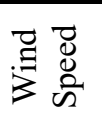 } & Mean & & 2.99 & 2.68 & 2.20 & 2.63 & 2.83 & 3.30 & 2.63 & 2.63 & 2.58 \\
\hline & STD & 1.25 & 1.47 & 1.55 & 1.27 & 1.41 & 1.51 & 2.02 & 1.41 & 1.41 & 1.33 \\
\hline \multirow{2}{*}{ 总兽 } & Mean & 123.38 & 64.31 & 71.92 & 74.85 & 38.63 & 70.83 & 61.40 & 38.63 & 38.63 & 45.08 \\
\hline & STD & 66.06 & 45.40 & 62.30 & 54.02 & 73.77 & 75.30 & 75.65 & 73.77 & 73.77 & 66.18 \\
\hline \multicolumn{2}{|c|}{ Night - $10 \mathrm{~m}$} & OBS & PBL1 & PBL2 & PBL3 & Met5 & Met6 & Init4 & Init5 & Hor5 & $\begin{array}{l}\text { Hor5- } \\
12 \mathrm{~km} \\
\end{array}$ \\
\hline \multirow{2}{*}{ 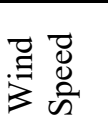 } & Mean & 2.25 & 2.81 & 2.58 & 2.18 & 2.51 & 2.72 & 2.91 & 2.51 & 2.51 & 2.66 \\
\hline & STD & 0.96 & 1.41 & 0.94 & 0.96 & 1.35 & 1.43 & 1.40 & 1.35 & 1.35 & 1.41 \\
\hline \multirow{2}{*}{ 劲 } & Mean & 222.98 & 244.07 & 243.95 & 263.07 & 226.97 & 230.93 & 160.02 & 226.97 & 226.97 & 295.43 \\
\hline & STD & 50.01 & 90.68 & 69.52 & 74.66 & 83.89 & 69.81 & 87.15 & 83.89 & 83.89 & 87.30 \\
\hline
\end{tabular}


Table S5 Summary of model performance in capturing wind speed and direction at BAO 300m during Aug 1-15, 2014

\begin{tabular}{|c|c|c|c|c|c|c|c|c|c|c|c|}
\hline \multirow{2}{*}{\multicolumn{2}{|c|}{ Day - 300 m }} & \multirow[b]{2}{*}{ OBS } & \multicolumn{3}{|c|}{ PBL } & \multicolumn{2}{|c|}{ Met } & \multicolumn{2}{|c|}{ init } & \multicolumn{2}{|c|}{ Horizontal Res. } \\
\hline & & & PBL1 & PBL2 & PBL3 & Met5 & Met6 & Init4 & Init5 & Hor5 & $\begin{array}{l}\text { Hor5- } \\
12 \mathrm{~km}\end{array}$ \\
\hline \multirow{2}{*}{ 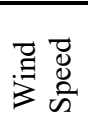 } & Mean & 3.23 & 3.89 & 3.51 & 2.78 & 2.88 & 3.22 & 3.83 & 2.88 & 2.88 & 2.77 \\
\hline & STD & 2.24 & 2.15 & 2.39 & 1.61 & 1.58 & 1.81 & 2.93 & 1.58 & 1.58 & 1.47 \\
\hline \multirow{2}{*}{ 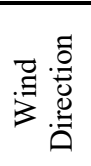 } & Mean & 117.31 & 62.69 & 62.42 & 64.05 & 32.91 & 57.67 & 56.71 & 32.91 & 32.91 & 39.52 \\
\hline & STD & 74.56 & 51.99 & 63.89 & 59.84 & 75.14 & 76.03 & 74.32 & 75.14 & 75.14 & 69.43 \\
\hline \multicolumn{2}{|c|}{ Night - 300 m } & & PBL1 & PBL2 & PBL3 & Met5 & Met6 & Init4 & Init5 & Hor5 & $\begin{array}{l}\text { Hor5- } \\
12 \mathrm{~km}\end{array}$ \\
\hline \multirow{2}{*}{ 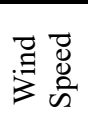 } & Mean & 3.42 & 5.00 & 4.34 & 3.80 & 4.21 & 4.60 & 5.07 & 4.21 & 4.21 & 4.89 \\
\hline & STD & 2.59 & 2.68 & 2.95 & 2.64 & 2.64 & 2.47 & 3.07 & 2.64 & 2.64 & 3.29 \\
\hline \multirow{2}{*}{ 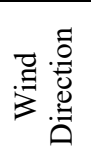 } & $\begin{array}{l}\text { Mean } \\
\text { Model }\end{array}$ & 213.59 & 141.12 & 223.36 & 355.95 & 326.05 & 294.02 & 156.88 & 326.05 & 326.05 & 306.58 \\
\hline & $\begin{array}{l}\text { STD } \\
\text { Model }\end{array}$ & 72.73 & 98.36 & 93.80 & 91.39 & 91.33 & 77.67 & 84.60 & 91.33 & 91.33 & 88.31 \\
\hline
\end{tabular}




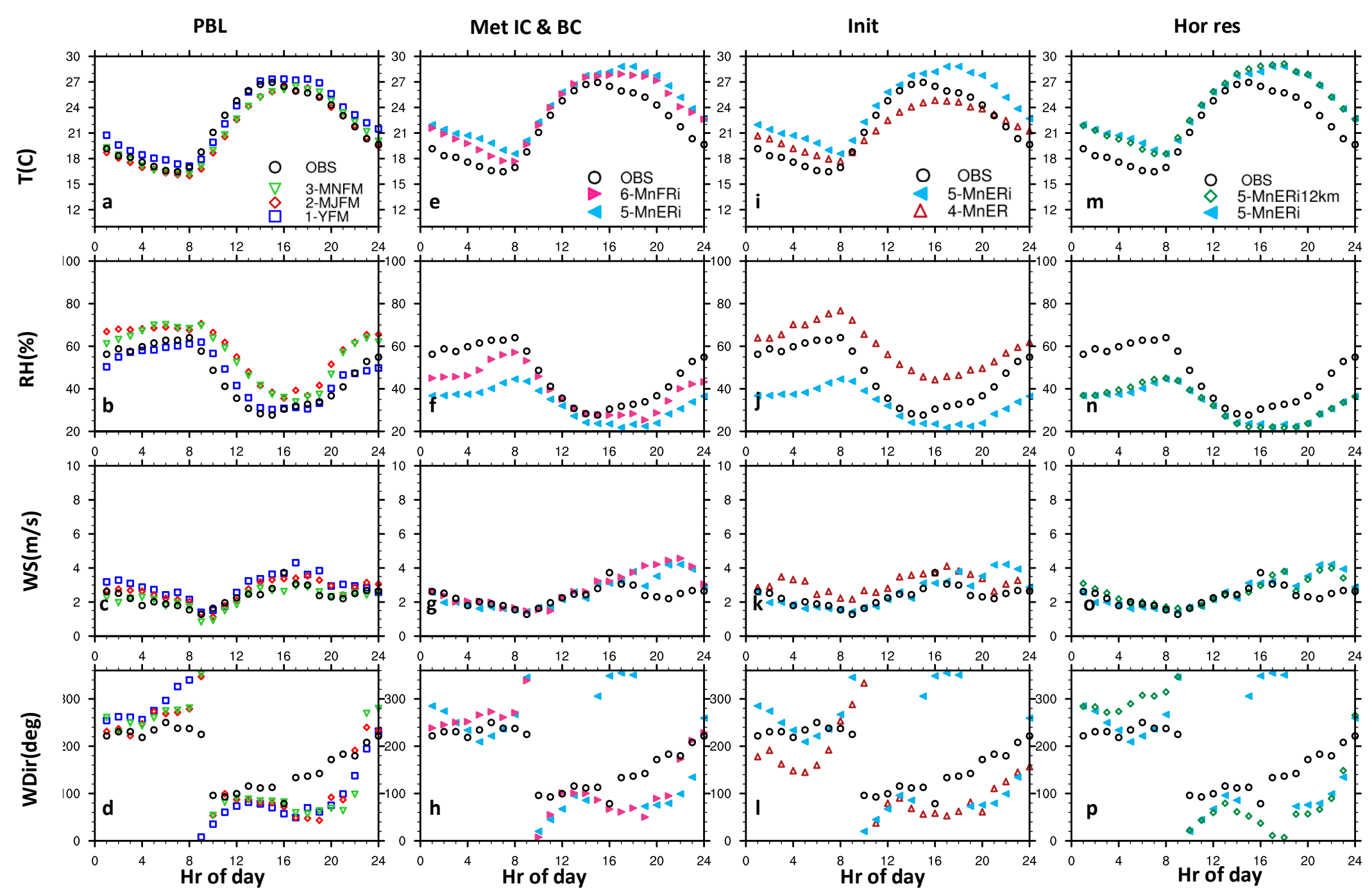

Figure S1 Average diurnal cycle of temperature, relative humidity, wind speed, and wind direction for all test sets and observation at BAO 10m. Averages are calculated for Aug 1 to 15, 2014 


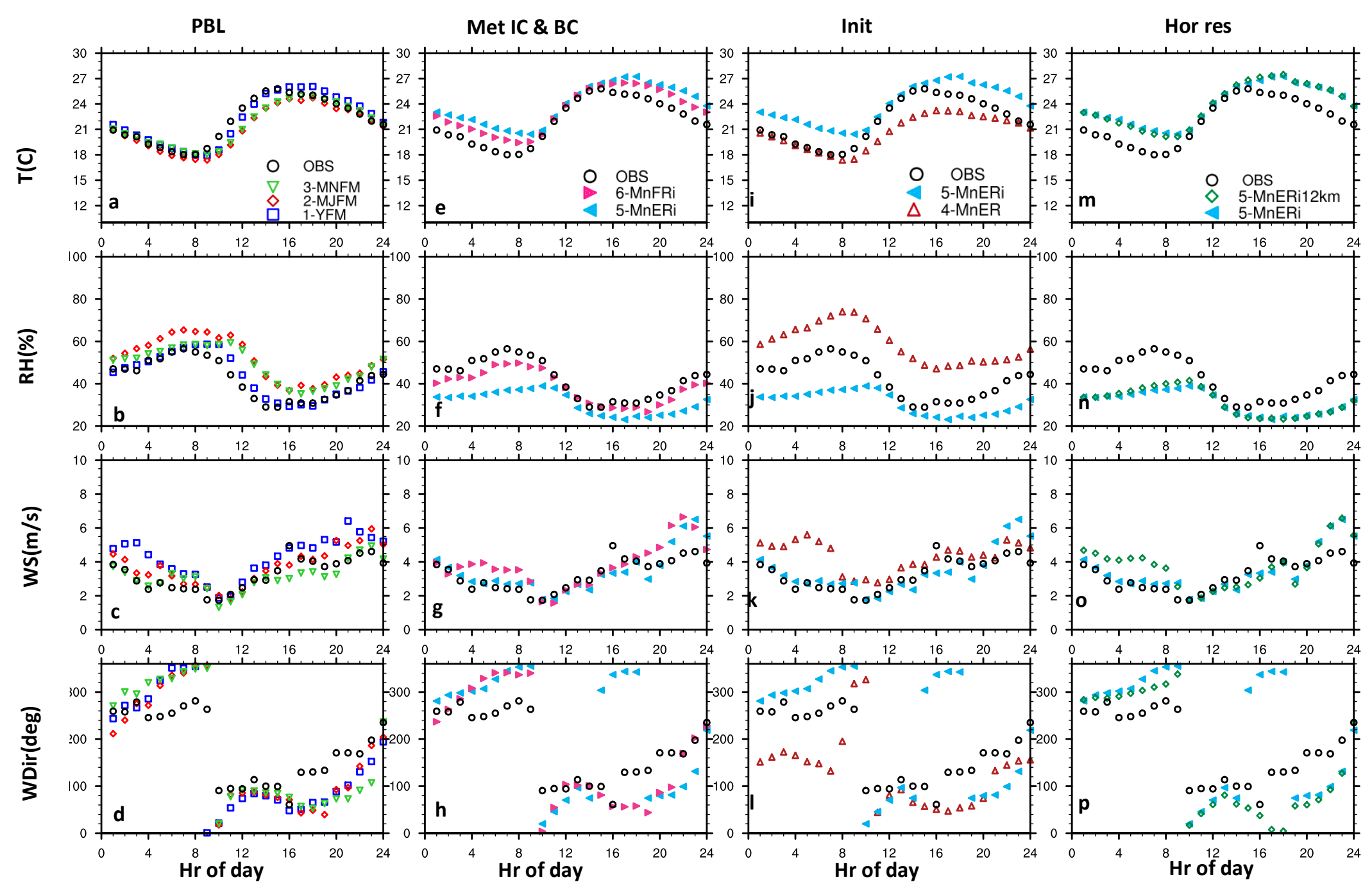

Figure S2 Average diurnal cycle of temperature, relative humidity, wind speed, and wind direction for all test sets and observation at BAO 100m. Averages are calculated for Aug 1 to 15, 2014 


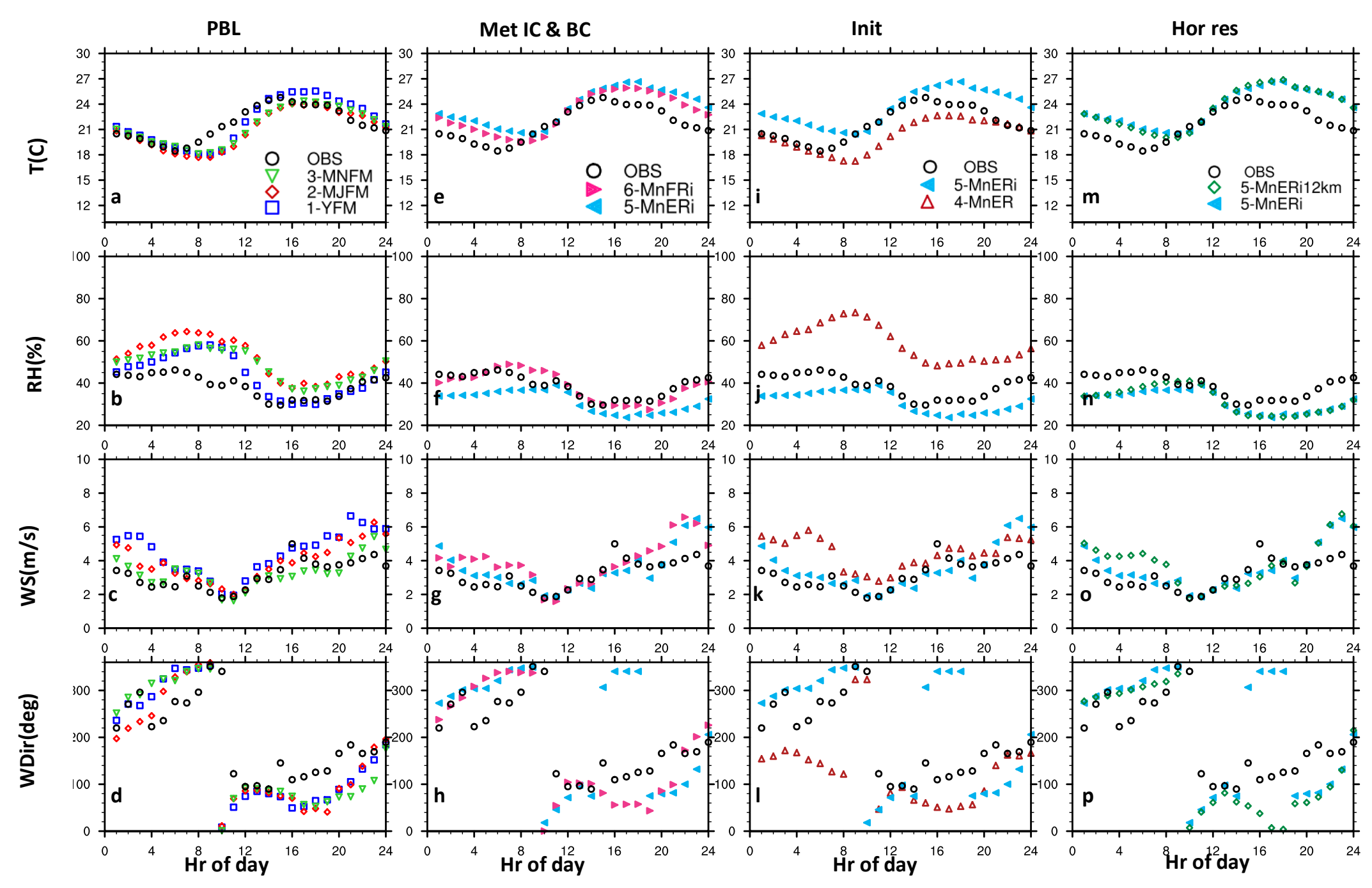

Figure S3 Average diurnal cycle of temperature, relative humidity, wind speed, and wind direction for all test sets and observation at BAO 300m. Averages are calculated for Aug 1 to 15, 2014 

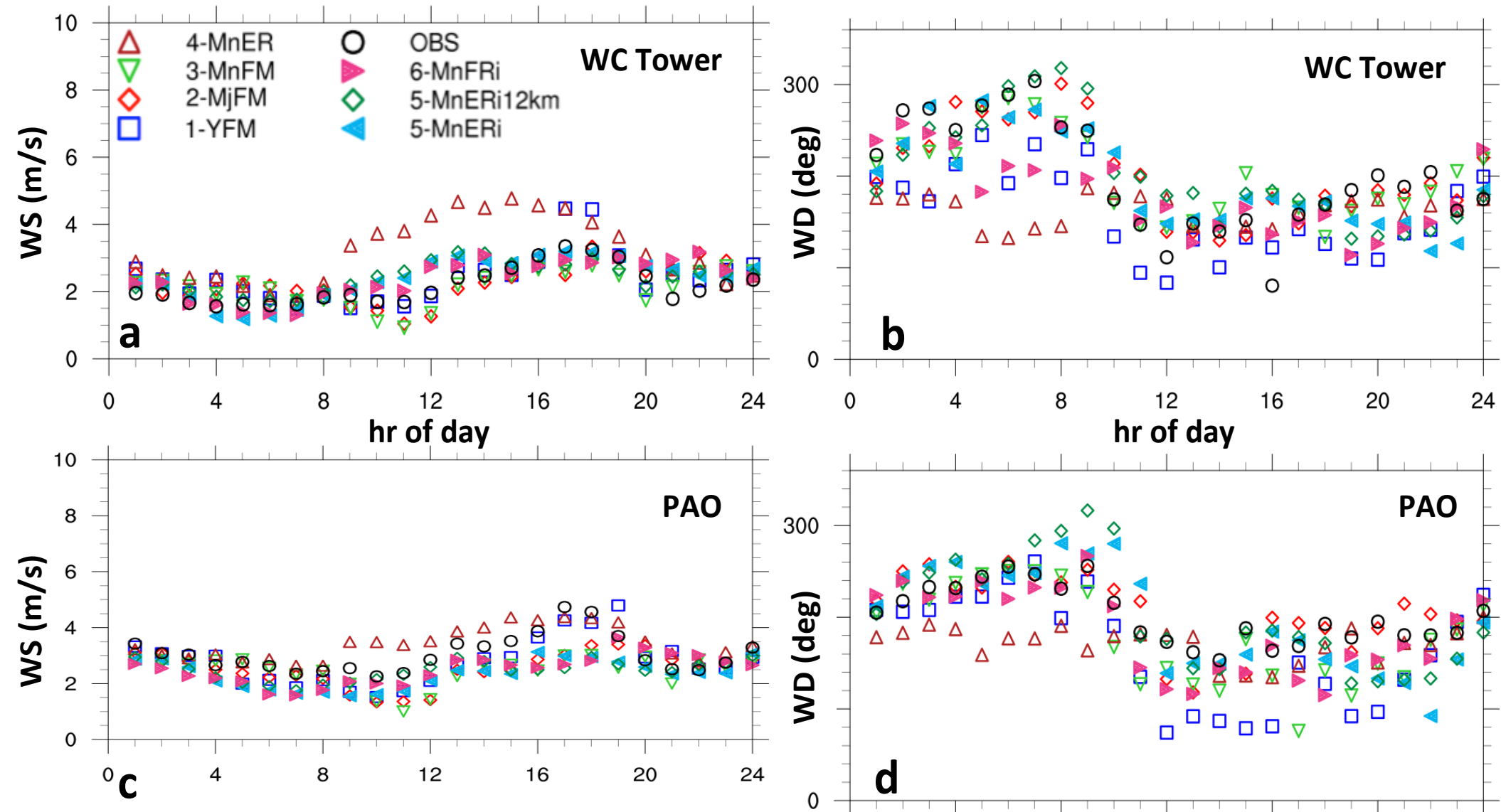

hr of day

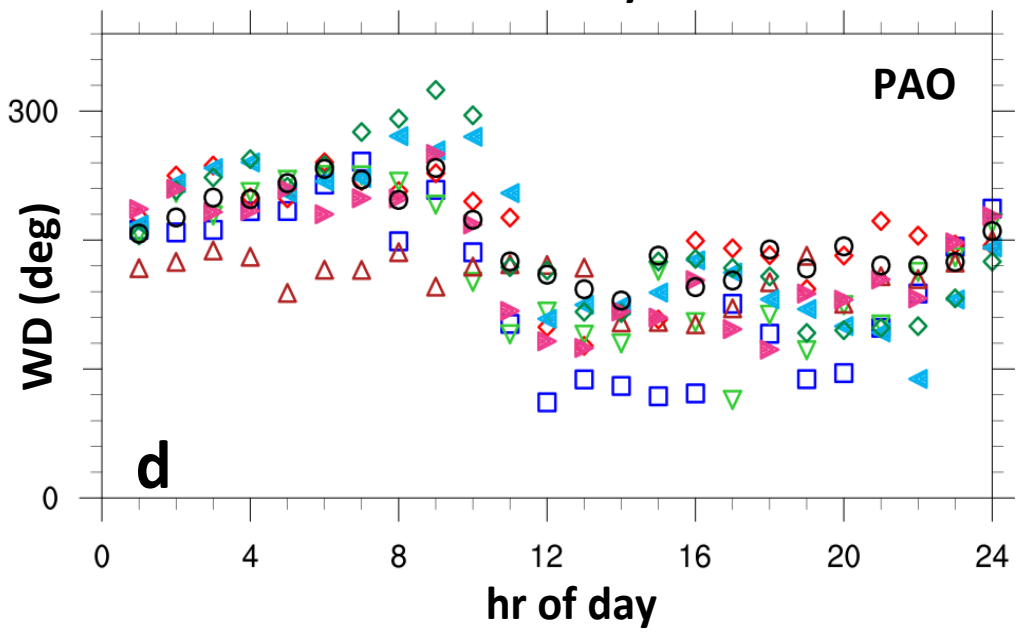

Figure S4. Average diurnal cycle of wind speed (WS) and direction (WD) at WC Tower and PAO sites. Averages are calculated for August 1 to 11, 2014 

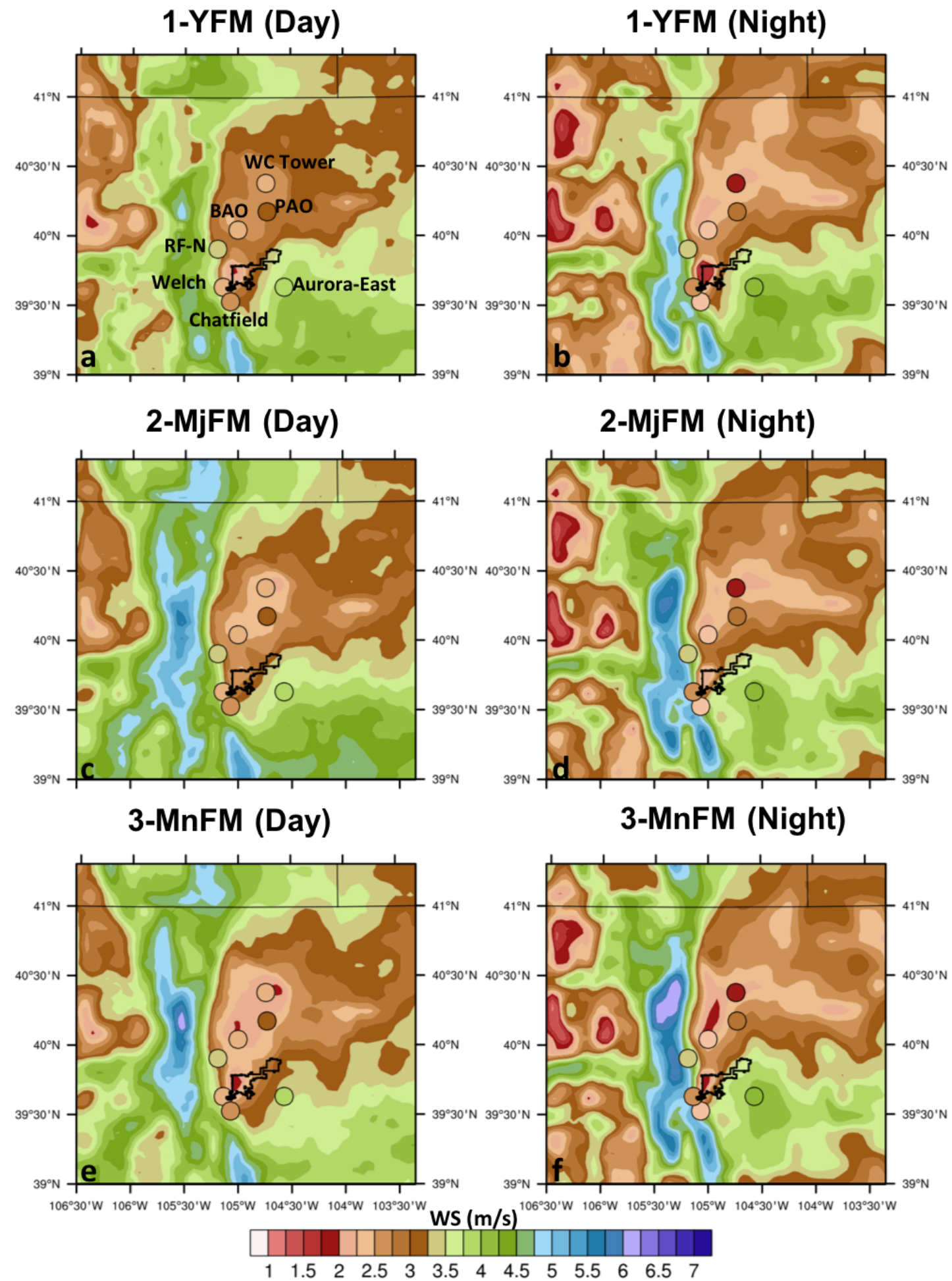

Figure S5 Wind speed at 10m captured by different PBL schemes. averaged from 1-August to 11-August 2014 

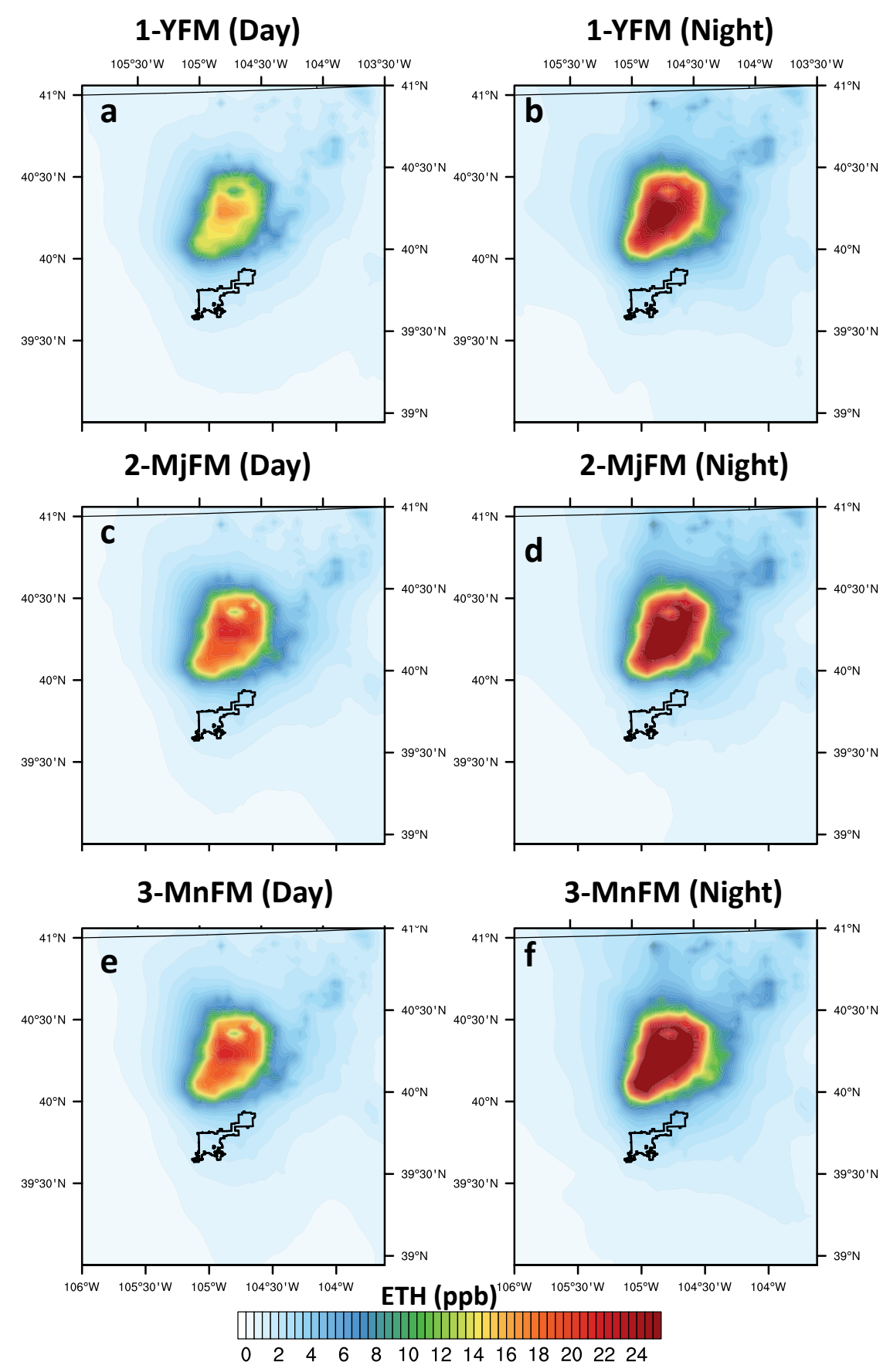

Figure S6. Surface ethane in sim 1 (1-YFM), sim 2 (2-MjFM), sim 3 (3-MnFm) averaged from August 1 to 15, 2014 

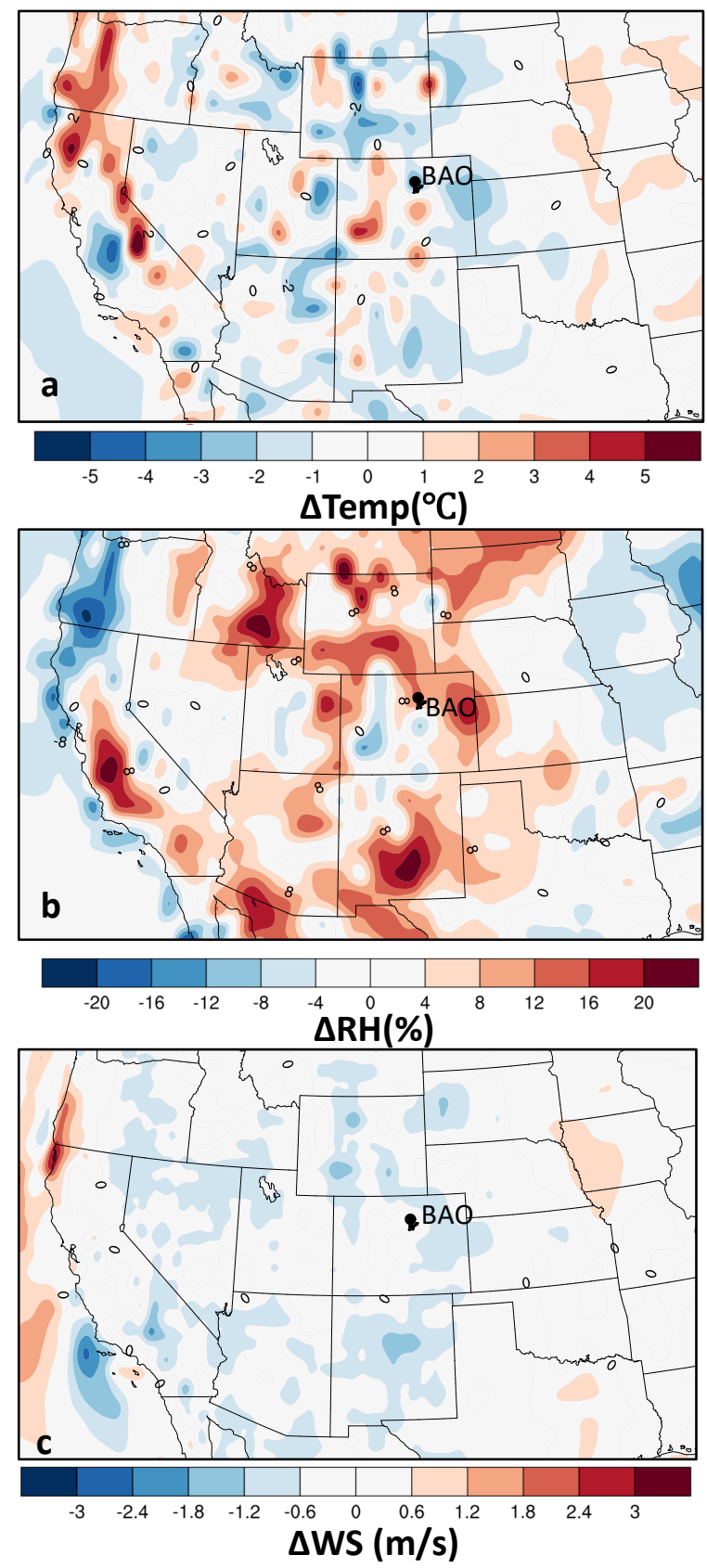

Figure S7 Differences in temperature (a), relative humidity (b), and wind speed (c) between ERA-interim and NCEP-FNL global models $(\Delta \mathrm{x}=\mathrm{X}($ ERA-interim $)-\mathrm{X}(\mathrm{NCEP}-\mathrm{FNL}))$ averaged from Aug 1 to 15, 2014 using 6-hourly data 

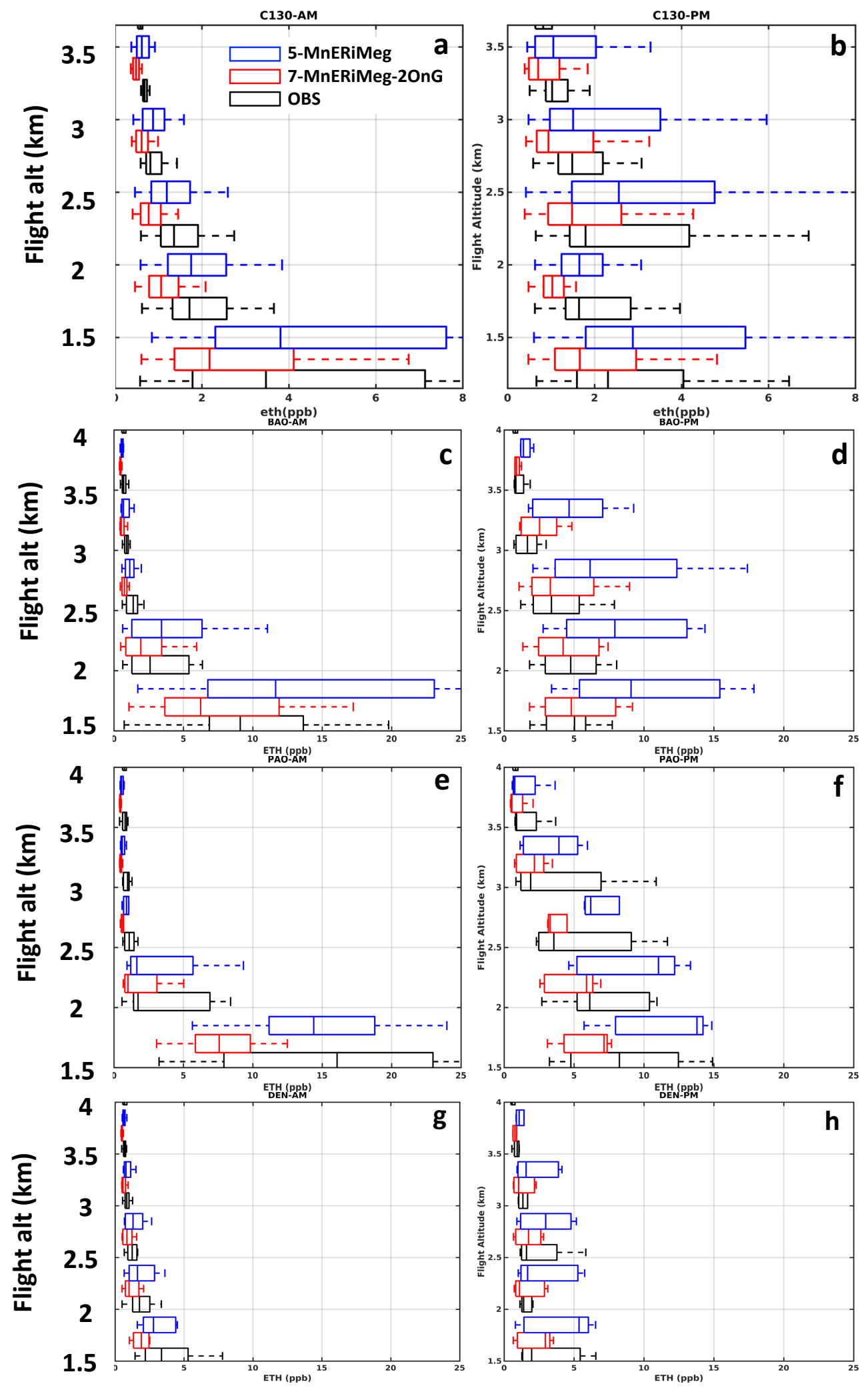

Figure S8. Sensitivity of ethane to oil and NG emission during C130-AM (a), C130-PM (b), P3-PAO AM (d), P3-PAO PM (c), P3-BAO AM (e), P3-BAO PM (f) averaged for August flights 